\title{
Proposal for a Chaotic Ratchet Using Cold Atoms in Optical Lattices
}

\author{
T. S. Monteiro, P. A. Dando, N. A. C. Hutchings, and M. R. Isherwood \\ Department of Physics and Astronomy, University College London, Gower Street, London WC1E 6BT, United Kingdom
}

(Received 5 April 2002; published 22 October 2002)

\begin{abstract}
We investigate a new type of quantum ratchet which may be realized by cold atoms in a double-well optical lattice, pulsed with unequal periods. The classical dynamics is chaotic and we find the classical diffusion rate $D$ is asymmetric in momentum up to a finite time $t_{r}$. The quantum behavior produces a corresponding asymmetry in the momentum distribution which is "frozen-in" by dynamical localization provided the break time $t^{*} \geq t_{r}$. We conclude that the cold atom ratchets require $D b / \hbar \sim 1$, where $b$ is a small deviation from period-one pulses.
\end{abstract}

DOI: 10.1103/PhysRevLett.89.194102

PACS numbers: 05.45.Mt, 05.40.Jc, 05.60.-k, 32.80.Pj

Cold atoms in optical lattices provide an excellent experimental demonstration of the phenomenon of $d y$ namical localization [1,2]. Dynamical localization (DL) has been described as the so-called "quantum suppression of classical chaos." In the usual realizations, a periodically driven or kicked system makes a transition to chaotic classical dynamics for sufficiently strong perturbation. The classical energy is unbounded and grows diffusively with time. For the corresponding quantum system, in contrast, the diffusion is suppressed after an $\hbar$-dependent time scale, the "break time" $t^{*}$. The final quantum momentum distribution is localized with a characteristic exponential profile. The formal analogy established with Anderson localization [2] forms a key analysis of this phenomenon. A series of recent experiments on cesium atoms in pulsed optical lattices [3] gave a classic demonstration of this effect.

The possibility of experiments with asymmetric lattices, in particular, with asymmetric double wells [4,5], leads us to investigate the possibility of constructing a "clean" atomic ratchet, where the transport results purely from the chaotic Hamiltonian dynamics, with no Brownian or dissipative ingredients. Ratchets are spatially periodic systems which, by means of a suitable spatialtemporal asymmetry, can generate a current even in the absence of a net force. There is already an extensive body of work on Brownian and deterministic ratchets with dissipation [6,7], driven by the need to understand biophysical systems such as molecular motors and certain mesoscopic systems. Some of this work encompasses the quantum dynamics [8]. For a full review see [9]. However, to date there has been very little work on Hamiltonian ratchets. One notable exception is the work by Flach et al. [10] where the general form of the spatial and temporal desymmetrization required to generate transport was investigated. The only substantial study of quantum Hamiltonian ratchets, however, is the work of Dittrich et al. [11] which showed how transport can occur in mixed phase spaces. They demonstrated that transport is zero if starting conditions cover all regions of phase space uniformly. A key result was a sum rule showing transport in the chaotic manifold is balanced by transport in the adjoining regular manifolds (stable islands/tori). Very recently [12], it was shown that a kicked map with a "rocking" linear potential leads to confinement in the chaotic region between a pair of tori which are not symmetrically located about $p=0$.

Here we propose a new type of Hamiltonian ratchet which, classically, is completely chaotic. This ratchet is not inconsistent with the rules established in [10,11], but has a quite different mechanism. It is the first example of a clean, nondissipative ratchet which is fully chaotic and, hence, does not require initial preparation on a specific set of islands/tori.

The basic mechanism relies on a hitherto unnoticed effect. In brief: consider particles in the asymmetric lattice, subject to a repeating cycle of kicks, of strength $K_{\text {eff }}$, perturbed from period one by a small parameter $b$. We find that the classical diffusion rates for positive and negative momenta $\left(D^{+}\right.$and $D^{-}$, respectively) are (in general) different up to a finite time, $t_{r}$. For $t<t_{r}$, an asymmetry in the classical momentum distribution $N_{\mathrm{cl}}(p)$ accumulates with kick number. Beyond this "ratchet" time $t_{r}$, the rates equalize, we have $D^{+} \sim$ $D^{-} \sim D$ (where $D \sim K_{\text {eff }}^{2} / 2$ is the total diffusion rate), and the net classical current $\left\langle p_{\mathrm{cl}}\right\rangle$ saturates to a constant, nonzero value. The energy, of course, continues to grow with time as $\left\langle p_{\mathrm{cl}}^{2}\right\rangle \sim D t$. The corresponding quantum current depends on $t^{*} / t_{r}$ : if the quantum break time is too short, no asymmetry in the quantum $N_{\mathrm{qm}}(p)$ accumulates and there is no quantum transport. If $t^{*} \gg t_{r}$, the localization length $L$ becomes large and the effective quantum momentum asymmetry $\sim\left\langle p_{\mathrm{qm}}\right\rangle / L$ decreases. We find that $t_{r} \sim \frac{1}{b^{2} D}$. A quantum ratchet will have the clearest experimental signature if $t^{*} \sim t_{r}$. Since $t^{*} \sim$ $D / \hbar^{2}$, the main conclusion of our Letter is that the cold atom ratchets need $D b / \hbar \sim 1$.

Consider the quantum dynamics for a Hamiltonian given by $H=\frac{p^{2}}{2}+K V(x) \sum_{n, i} \delta\left(t-n T_{i}\right)$. The ratchet potential is given by $V(x)=\{\sin x+a \sin (2 x+\Phi)\}$. In the usual realization of DL, the quantum kicked rotor $(\mathrm{QKR})$, the kicks are equally spaced. For the ratchet, 
they are unequal but we can use a rescaled time such that, without loss of generality, we take $\left\langle T_{i}\right\rangle=1$ over each cycle. We take a repeating cycle of "chirped" kick period spacings, $1+j b, 1+(j-1) b \ldots, 1-(j-1) b, 1-j b$ where $j>0$ is an integer and $b$ is a small time increment. $N=2 j+1$ for $N$ odd and $N=2 j$ for $N$ even. Then, our $N=3$ cycle corresponds to a repeating set of kick spacings $T_{1}=1+b, T_{2}=1, T_{3}=1-b$ while an $N=2$ cycle corresponds to $T_{1}=1+b, T_{2}=1-b$, and so forth.

The time-evolution operator for the $i$ th kick of the $n$th cycle factorizes into a free and a "kick" part $U_{i}=$ $U_{i}^{\text {free }} U^{\text {kick }}$. In the usual plane wave basis, for a given quasimomentum $q$, the matrix elements of $U_{i}$ can be shown to take the form:

$$
U_{m l}^{(i)}(q)=e^{-i\left[\hbar T_{i}(l+q)^{2} / 2\right]} \sum_{s} e^{i s \Phi} J_{l-m-2 s}\left(\frac{K}{\hbar}\right) J_{s}\left(\frac{a K}{\hbar}\right),
$$

where the $J$ are ordinary Bessel functions. The timeevolution operator for one period $U\left(T=\sum_{i} T_{i}\right)=$ $\prod_{i=1}^{i=N} U_{i}$. In the experiments, an important parameter is an effective $\hbar_{\text {eff }}=8 \omega_{r} T$, where $\omega_{r}$ is the recoil frequency. In [3], $\hbar_{\text {eff }} \sim 1$, so here we have considered the range $\hbar=1 \rightarrow 1 / 10$.

In Fig. 1 we compared the evolution of a quantum wave packet with equal kick times $\left(T_{i}=1\right)$ with a correspond-

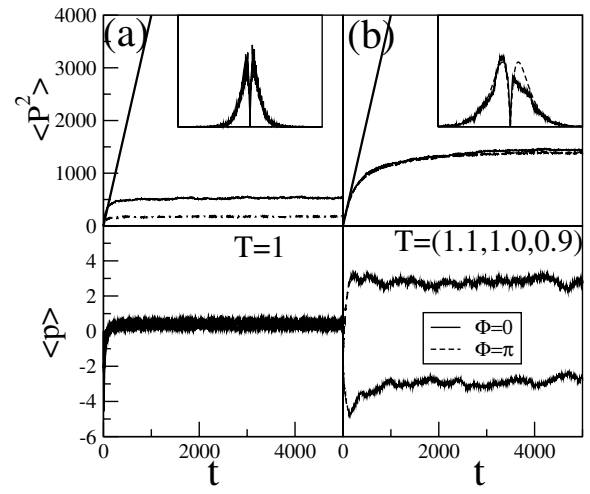

FIG. 1. Effect of equal and unequal kick spacings on a minimal uncertainty Gaussian wave packet with $\hbar=1 / 2$ with initial $\langle p\rangle=0$, for kick strength $K=2$ and $a=1 / 2$, for different $\Phi$. (a) Evolution of energy and momentum for $T=1$ (dw-QKR). (b) Evolution of energy and momentum for a repeating $N=3$ cycle of kicks with $b=0.1$, hence $T_{i}=$ 1.1, 1.0, 0.9 (cdw-QKR). (a) and (b) show that there is no transport for the equal kick case, but that there is a substantial net momentum ( $\sim$ constant if $t>t^{*}$ ) for the cdw-QKR. Setting $\Phi=\pi$ reverses the current relative to $\Phi=0$. The insets show the first moment of the $p$ distribution: The current is the difference in area between the positive $p$ and negative $p$ "bumps." The DL form $|p| \cdot N(|p|)=\frac{|p|}{2 L} \exp -|p| / L$ [with $L=$ $27.5 \sim 3.5 D_{0} / \hbar$ (see Fig. 3)] is superposed, showing the DL form is regained for large enough $p$. ing unequal-kick case with $N=3, b=0.1$. Since $V(x)$ in general represents a double-well potential, we refer to it as the dw-QKR to distinguish it from the standard map case with $V=K \sin x$. We refer to the unequal-kick case as the chirped or cdw-QKR.

The upper graph in Fig. 1 shows that, in both cases, the average quantum energy initially increases linearly, i.e., $\left\langle p^{2}\right\rangle \sim D t$. The straight line corresponding to the classical energy is also shown. Neglecting all classical correlations, we find $D \sim D_{0}=K_{\text {eff }}^{2} / 2$, where $K_{\text {eff }}=$ $K \sqrt{\left(1+4 a^{2}\right)}$. The $\Phi$ dependence lies in neglected correlations, which in this case appear as products of Bessel functions [13]. However, beyond a time scale $t \sim t^{*}$, the quantum energy saturates to a constant value $\left\langle p^{2}\right\rangle \sim L^{2}$, where $L$ is the localization length.

The dw-QKR shows a typical, exponentially localized, momentum distribution. However, for the cdw-QKR, the quantal $N(p)$ is evidently asymmetric, but regains the DL form at large $p$. There is no net current in the $T=1$ case: asymptotically, $\langle p\rangle \simeq 0$. However, for the chirped case, for $t>t^{*}$, in general we have $\langle p\rangle \sim$ constant. A meaningful way to quantify the asymmetry is a rescaled momentum $p_{L}=\langle p\rangle / L$ which also tends to a constant for $t>t^{*}$ [e.g., $p_{L} \simeq 1 / 8$ in Fig. 1(b) for $\Phi=0$ ]. Taking $\Phi=\pi$ reverses the symmetry of $V(x)$ and the direction of motion relative to $\Phi=0$. Intermediate values of $\Phi$ typically give $\langle p\rangle$ within these extremes.

In summary, the cdw-QKR shows a ratchet effect and dynamical localization, with an asymmetric profile at short range, but a DL form for large $p$. The quantal current increases from zero for a finite time, then saturates to constant magnitude. This is surprising, since it is thought that in the fully chaotic regime a Hamiltonian ratchet current should not persist. In order to understand this behavior, we now compare with the corresponding classical current.

In Figs. 2(a) and 2(b) we show that, in fact, both quantum and classical currents $\langle p\rangle$ increase in magnitude, then saturate to a constant value after a finite time; but the saturation time is generally different: $t^{*}$ in the quantum cases in Fig. 2(a) and another, ratchet time scale $t_{r}$ in the classical cases shown in Figs. 2(a) and 2(b). Figure 2(a) shows, in particular, the dependence on $\hbar$. The $\left\langle p_{\mathrm{qm}}\right\rangle$ are negligible for $\hbar>1$ but increase rapidly with decreasing $\hbar$, up to $\hbar \sim 0.25$. This is important for any experiment : for these parameters $(D \sim 2.5$, $b=0.1$ ), an experiment with $\hbar_{\text {eff }} \sim 0.8$ would show little asymmetry, but just halving $\hbar_{\text {eff }}$ to $\sim 0.4$ would show substantial asymmetry. Beyond $\hbar \sim 0.4,\left\langle p_{\mathrm{qm}}\right\rangle$ is comparable to the saturated classical value. But since the most experimentally "detectable" ratchet is one which maximizes the asymmetry $N(p)$, this means maximizing a rescaled momentum $p_{L}=\left\langle p_{\mathrm{qm}}\right\rangle / L$, so there is no advantage in reducing $\hbar$ much below $\sim 0.4$ since $L \sim \hbar^{-1}$. Figure 2(b) shows that, for a given $b \quad(b=0.05$ in this graph), the classical saturation time $t_{r}$ falls with 


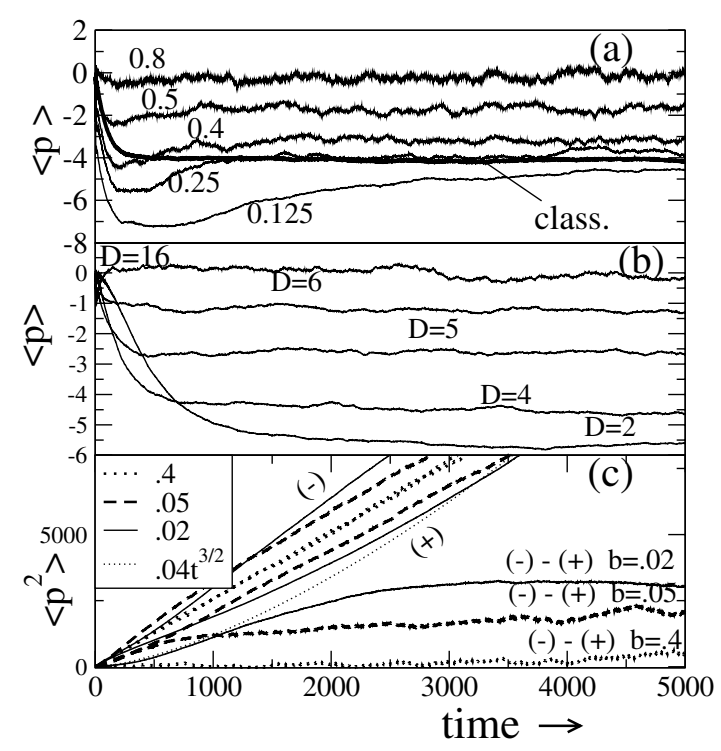

FIG. 2. Quantal versus classical ratchet currents are compared in (a) and (b) for $N=3$. Both the classical and quantum $\langle p\rangle$ reach a constant value after a finite time $\left(t^{*}\right.$ and $t_{r}$, respectively). (a) The quantum current is very sensitive to $\hbar$ if the quantum (Gaussian) wave packet localizes before the classical equivalent saturates. The values of $\hbar,(0.8 \rightarrow 0.125)$ are indicated. $K=1.6, a=0.5$ (hence, $D_{0} \sim 2.5$ ), and $b=0.1$. The asymptotic quantum $\langle p\rangle$ increases with $\hbar$ and "catches" up with the classical results for $\hbar \sim 0.25$ where $t^{*} \sim t_{r}$. (b) Evolution of $\langle p\rangle$ for a classical "wave packet" (500 000 particles with a Gaussian random distribution in $x$, $p$, of width $\sigma=1.5$ for $b=0.05$ but different $D)$. The current increases and then saturates for $t>t_{r} \sim 1 /\left(D b^{2}\right)$. (c) Differential classical diffusion rates $\left[D^{+}(t, b), D^{-}(t, b)\right]:\left\langle p^{2}\right\rangle$ is evaluated separately at each kick for particles with positive and negative momenta for $K=1.6, a=0.5, D_{0} \sim 2.5$, and different $b$. We see that $\left\langle p^{2}\right\rangle^{ \pm}$diverge from linear growth by a quantity, which is similar in magnitude but opposite in sign for the negative and positive components. The + and - indicate $\left\langle p^{2}\right\rangle^{+},\left\langle p^{2}\right\rangle^{-}$, respectively. But once $t>t_{r}$, we see linear growth: $D^{+} \sim$ $D^{-} \sim D \sim 2.5$. The lower graphs show $\delta(b)=\left\langle p^{2}\right\rangle^{(-)}-$ $\left\langle p^{2}\right\rangle^{(+)}$(for these parameters, $D^{-}>D^{+}$so the current is negative). $\delta(b) \propto b t^{3 / 2}$ for small $t$.

increasing $D$. The fact that $\left\langle p_{\mathrm{cl}}\right\rangle$ saturates at all is surprising: after all, the ensemble of classical trajectories is continually expanding and exploring new phase-space regions corresponding to higher momenta.

While $t^{*}$ is well studied, $t_{r}$ is quite new; we find that it can be understood in terms of differential rates of absorption of energy for particles moving left or right. To illustrate this, we calculated-separately $-\left\langle p^{2}\right\rangle^{(-)}$for those particles with $p<0$ and $\left\langle p^{2}\right\rangle^{(+)}$for those with $p>0$. The results are shown in Fig. 2(c) for $D_{0} \sim 2.5$ and different $b$. They are quite striking: $\left\langle p^{2}\right\rangle^{(-)}$and $\left\langle p^{2}\right\rangle^{(+)}$separate gradually, more or less symmetrically, about the line $\sim 2.5 t$, but beyond a certain time, they run parallel to each other and their slopes become equal with $D^{+} \sim D^{-} \sim 2.5$. Also in Fig. 2(c) we plot $\delta(b)=$ $\left\langle p^{2}\right\rangle^{(-)}-\left\langle p^{2}\right\rangle^{(+)}$for each $b$ since this shows the saturation effect more clearly. We find numerically and from theory that, for small $t, \delta(b) \simeq b\left(D_{0} t\right)^{3 / 2} f(K, a)$, where $f(K, a)$ is a function of $K, a$.

The so-called "quasilinear" approximation for the energy diffusion $D_{0} \simeq K^{2} / 2$ [14] neglects correlations between sequences of consecutive kicks; for the standard map, these give well known corrections to the diffusion constant in the form of Bessel functions: $D=$ $\frac{K^{2}}{2}\left\{1-2\left[J_{1}(K)\right]^{2}-2 J_{2}(K) \ldots\right\}[14,15]$. These corrections have even been measured experimentally with cold cesium atoms in pulsed optical lattices [16,17]. For example, the $2 J_{2}(K)$ term originates from two-kick correlations of the form $C(2)=2\left\langle V^{\prime}\left(x_{i}\right) V^{\prime}\left(x_{i+2}\right)\right\rangle$. In general, if we work out the change in $\left\langle p^{2}\right\rangle$ for successive kicks, we obtain a diffusion rate $D$ which is the same whether we average the momentum from $0 \rightarrow \infty$ or from $0 \rightarrow-\infty$. Odd terms in $p$ such as $\langle\sin 2 p\rangle$ average to zero once the cloud has an appreciable spread, while the even terms $\left\langle\sin ^{2} p\right\rangle=$ $\left\langle\cos ^{2} p\right\rangle \simeq 1 / 2$ are insensitive to the sign of $p$ : in the standard map, $D^{+}=D^{-}$. But if we consider the first kick of the $n$th cycle of the cdw-QKR, we have:

$$
\begin{gathered}
x_{n 1}=x_{n 0}+P_{n 0}(1+b), \\
P_{n 1}=P_{n 0}-K V^{\prime}\left[x_{n 0}+P_{n 0}(1+b)\right] .
\end{gathered}
$$

The unequal kicks allow free evolution for an additional small distance $\delta_{1}=P_{n 0} b$. To first order,

$$
P_{n 1}=P_{n 0}-K\left\{V^{\prime}\left(x_{n 0}+P_{n 0}\right)+P_{n 0} b V^{\prime \prime}\left(x_{n 0}+P_{n 0}\right)\right\} .
$$

Hence, we now have correlations which depend on the sign of $p$ and which scale with $b$. More precisely, we have averages of typical form $I(p)=\left\langle\sin p b \sin ^{2} p\right\rangle$. For $p b$ small, $I(p) \simeq\left\langle p_{ \pm}\right\rangle b / 2 \simeq \pm \frac{b}{2} \sqrt{D_{0} t}$ when we average positive and negative momenta separately. The average over $x$ yields a function $f(K, a)$. Hence, clearly the corresponding energy $\left\langle p^{2}\right\rangle^{ \pm} \sim D_{0} t \pm\left(D_{0} t\right)^{3 / 2} b f(K, a) . f(K, a)$ is a very complicated function involving sums of products of Bessel functions [13], but yields good estimates of $\delta(b)$. In [18] we have investigated the rocking ratchet of [12] in the chaotic regime and found the same physics. We obtained excellent agreement between a curve $\propto b t^{3 / 2}$ and numerics and can predict successfully repeated current reversals without any detailed study of phase space.

For $p b$ large, $I=\left\langle\sin p b \sin ^{2} p\right\rangle \simeq 0$, so there is no ratchet correction. The associated saturation time $t_{r}$ is very important since then the classical ratchet speed reaches its maximum and for $t>t_{r},\left\langle p_{\mathrm{cl}}\right\rangle \sim c s t$. We identify it as a point where $I \sim 0$; hence, we take $b \sqrt{\left\langle p^{2}\right\rangle^{(+)}} \sim \pi$ for the positive component and $b \sqrt{\left\langle p^{2}\right\rangle^{(-)}} \sim \pi$ for the negative component. For an order of magnitude estimate of the mean time scale involved, we take $b \sqrt{D t} \sim \pi$. Hence, we obtain $t_{r} \sim \frac{\pi^{2}}{D b^{2}}$. In [12] 


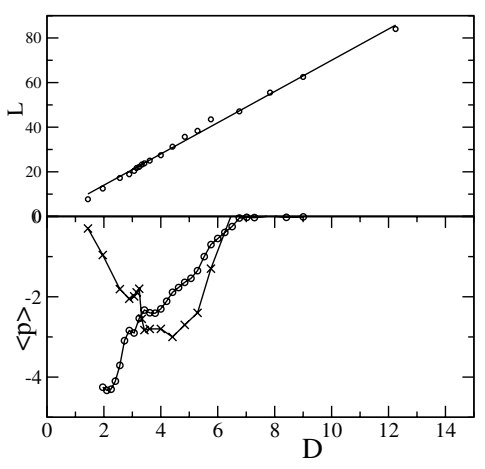

FIG. 3. (a) Relation between classical diffusion rate $D$ and the quantum localization length $L$ for the cdw-QKR. The solid line corresponds to $L=\frac{3.5 D}{\hbar}$. (b) Current against $D$ for a quantum wave packet (crosses) and a classical "Gaussian wave packet" (circles). The graph illustrates the fact that if the quantum break time is too short (low $D$ ) the quantum momentum is small, but catches up with the classical momentum at $t^{*} \sim t_{r}$.

a long-ranged periodicity in $p$, of order $2 \pi / b$, was identified. We note that for our study of systems with zero initial current, this is not significant, since by the time the periodicity boundary is sampled $I(p) \simeq 0$. However, it is an important consideration if we start with nonzero initial current [18].

Numerically, we estimated $t_{r} \sim \frac{5}{D b^{2}}$ which is not inconsistent with the above. This explains the counterintuitive behavior that the larger deviation from period-one kicking (i.e., the larger $b$ ) give a smaller ratchet effect. Though the perturbation scales as $b$, the time for which it is important scales as $b^{-2}$.

For the standard map/QKR, there is a well known relation between the quantum localization length and the classical diffusion constant: $L \sim \frac{\alpha D}{\hbar}$, where the constant $\alpha$ was found to be $1 / 2$ [15]. The $N=3, b=0.1$ cdw-QKR takes a modified proportionality constant, i.e., $L \sim \frac{3.5 D}{\hbar}$.

In Fig. 3(a) we plot a set of calculated $L$ (which range from $L \sim 10-80$ ) against $D$ for $\hbar=1 / 2$ together with the line corresponding to $L=\frac{3.5 D}{\hbar}$. The agreement is excellent. From $L^{2} \sim D t^{*}$ we obtain $t^{*} \sim 12 D / \hbar^{2}$.

In Fig. 3(b), for the quantum distributions in Fig. 3(a), we have also plotted the current as a function of $D$, together with their classical equivalents, obtained from an ensemble of 500000 classical particles. We see that the classical $\langle p\rangle$ fall monotonically with $D$, apart from fine structure which we attribute to cantori. The quantum results, however, for low $D$ are much smaller than the classical values but increase in magnitude until there is a "crossover" point at $D \sim 3$, after which they are much closer to the classical values.

We do not expect perfect agreement with the classical results for $\hbar=1 / 2$; a cleaner comparison might be ob- tained for smaller $\hbar$, but this might be harder to achieve in an experiment.

We estimate the quantum break time at the crossover $t^{*} \sim 12 D / \hbar^{2} \sim 150$ kicks. The ratchet time $t_{r} \sim 5 /$ $\left(D b^{2}\right) \sim 160$ kicks. Such good agreement is somewhat fortuitous, since there are larger uncertainties in the time scales. Nevertheless, it does provide us with a useful guide for the best parameters for an experiment.

So one of our key results is that the requirement $t^{*} \sim t_{r}$ implies that we need $D b / \hbar \sim 1$. The $L$ values should, of course, be experimentally plausible $(L \sim 10-100)$, so this places a constraint on $D / \hbar$.

In conclusion, we have proposed a mechanism for the first generic, completely chaotic Hamiltonian ratchet.

We thank Professor S. Fishman for helpful advice. The work was supported by EPSRC Grant No. GR/N19519.

[1] G. Casati, B. V. Chirikov, F. M. Izraelev, and J. Ford, in Stochastic Behavior in Classical and Quantum Hamiltonian Systems, Lecture Notes in Physics Vol. 93 (Springer, Berlin, 1979), p. 334.

[2] S. Fishman, D. R. Grempel, and R. E. Prange, Phys. Rev. Lett. 49, 509 (1982).

[3] F. L. Moore, J. C. Robinson, C. F. Bharucha, B. Sundaram, and M. G. Raizen, Phys. Rev. Lett. 75, 4598 (1995).

[4] C. Mennerat-Robilliard et al., Phys. Rev. Lett. 82, 851 (1999).

[5] We thank Dave Meacher for explaining possible experiments with driven double-well lattices.

[6] F. Julicher, A. Adjari, and J. Prost, Rev. Mod. Phys. 69, 1269 (1997).

[7] J. L. Mateos, Phys. Rev. Lett. 84, 258 (1999).

[8] P. Reimann, M. Grifoni, and P. Hänggi, Phys. Rev. Lett. 79, 10 (1997); P. Reimann and P. Hänggi, Chaos 8, 629 (1998).

[9] P. Reimann, Phys. Rep. 361, 57 (2002).

[10] S. Flach, O. Yevtushenko, and Y. Zolotaryuk, Phys. Rev. Lett. 84, 2358 (2000).

[11] T. Dittrich, R. Ketzmerick, M.-F. Otto, and H. Schanz, Ann. Phys. (Berlin) 9, 755 (2000); H. Schanz, M.-F. Otto, R. Ketzmerick, and T. Dittrich, Phys. Rev. Lett. 87, 070601 (2001).

[12] T. Cheon, P. Exner, and P. Seba, cond-mat/0203241.

[13] N. Hutchings et al. (to be published).

[14] A. J. Lichtenberg and M. A. Lieberman, Regular and Chaotic Dynamics (Springer-Verlag, New York, 1992).

[15] D. L. Shepelyansky, Phys. Rev. Lett. 56, 677 (1986).

[16] B. G. Klappauf, W. H. Oskay, D. A. Steck, and M. G. Raizen, Phys. Rev. Lett. 81, 4044 (1998).

[17] M. B. d'Arcy, R. M. Godun, M. K. Oberthaler, D. Cassetari, and G. S. Summy, Phys. Rev. Lett. 87, 074102 (2001).

[18] M. R. Isherwood and T. S. Monteiro, physics/0206072. 